\title{
Mitotic Cell Studies Based on in vivo Observations XI. Various causes which prevent an elucidation of mitosis
}

\author{
Bungo Wada ${ }^{1}$ \\ Home address: 15-1, Kamiyama-cho, Shibuya-ku, Tokyo, 150 Japan
}

Received December 2, 1979

From the results of in vivo observations and experiments carried out mainly on the mitosis of living cells in Tradescantia staminal hair cells, the author has reached a conclusion that karyokinesis is performed according to a common principle in both plant and animal cells irrespective of whether they are of the higher or lower eucaryotes (Wada 1977).

This principle has been understood by a few plant cytologists but disregarded by animal cytologists. This reason is very clear. This principle has no need for centrioles in the chromosome movement in anaphase as animal cytologists believe. Based on the atractoplasm theory (Fujii 1931) and the birefringent figures of spindles in living plant and animal cells (Inoué 1953), the author has taken into consideration the following new findings and principles which have brought epoch-making advances in biology in the later half of this century. There are 1) the existence of the spindle membrane, (an intracellular biological membrane); 2) the analysis of the behavior of centrioles, astral rays and karyokinetic spindles according to the one gene-one enzyme theory (Beadle and Tatum 1941);3) the increase of gene DNA (chromosomes) in the course of evolution in eucaryotes; 4) gene control for the structure and function of mitotic spindles and 5) teleonomy in the manifestation of gene activity (Monod 1970). However, cytologists working on mitosis are practically indifferent to these new findings mentioned above. Animal cytologists seem concerned very little with the contributions which have developed in the field outside of fixation cytology (the latter including electron microscope studies).

The subject of mitosis has been studied by hundreds of cytologists since the establishment of cytology but no sufficient interpretations has yet been achieved. This failure may be due fundamentally to a lack of knowledge by cytologists about the true situation concerning the differences between routine demonstrations of mitosis (discontinuous figures obtained from fixed cells) and the actual evidence (continuous behavior of dividing cells traced by in vivo observations).

In this paper the author will point out various interpretations of mitosis commonly accepted and described in cytology books which have been precluding an elucidation of mitosis from the introduction of the new findings and principles in the modern biological sciences. 
1. Intrinsic difference between mitotic spindles observed in fixed cells and those in living cells

Brilliant contributions in biology achieved in the later half of this century are based on observations, experiments or analysis carried out on research material; Monod (1970) stated "The postulate of objectivity is consubstantial with sciences".

To grasp the continuous changes of cells in mitosis objectively however, there is no other means than in vivo observations. Since the establishment of fixation techniques in cytology, it is a settled custom and authorization to consider fixed figures as if they were facts in study on cytology. In this way of thinking and with these techniques in cytology, thousands of contributions have brought success to studies on static morphology, karyology, cytogenetics, histology, anatomy and to many other field of biology, agriculture and medicine. However, this commonly held idea in fixation cytology is not always true nor a contribution to the study of the continuous changes of dynamic cytomorphology in living mitotic cells.

\section{1) Spindle figures observed in fixed mitotic cells}

Fixed cells in metaphase contain granules in various sizes (plastids, mitochondria, cytoplasmic granules or centrioles and astral rays in animal cells). They appear around spindles but no membraneous structures are found which envelope spindles.

As a result of such a distribution of granules in fixed mitotic cells, cytology books state that the nuclear membrane breaks down before spindle formation. This interpretation of the nuclear membrane is an indirect demonstration inductively accepted from thousands of fixed mitotic cells observed by cytologists since the establishment of cytology, but not an actual evidence confirmed in living mitotic cells objectively by means of in vivo observations.

2) Spindle figures observed in living mitotic cells

A living cell which enters mitosis continuously shows the following morphological changes under in vivo observations: 1) The nucleus increases its volume remarkably during prophase and transforms its outline from sphere to spindle-form. 2) Surrounding the nucleus, many granules in various sizes show Brownian movement, or a streaming in the cytoplasm, but none of them enter into the spindle area. This behavior of the granules in living mitotic cells is interpreted in physics as due to the spindle being enveloped with a membrane (an intracellular biological membrane), provided the spindle body would not be a solid body.

From the results of in vivo observations on mitotic cells, it is logical to conclude that the nuclear membrane of the metabolic nucleus continuously transforms itself into a spindle membrane. If nuclear membranes were to break down and disappear before spindle formation as fixation cytologists believe, an explosive shock would occur at the moment of membrane destruction accompanied by a movement or streaming of the granules in the cytoplasm, but no cytologists seem to have ever seen such catastrophic phenomena in cine-microfilms of mitosis in plant and animal cells. 
The idea that the breakdown of the nuclear membrane before spindle formation described in cytology books seems to be the greatest and most fundamental error to impede an correct interpretation of mitosis.

2. The chromosome movement in anaphase is nothing but a separation of sister chromosomes to form daughter nuclei

The chromosome movement in anaphase is, at present, treated as an insoluble problem by animal cytologists. It seems the fashion to discuss the structure and function of the so-called mitotic apparatus by taking centrioles, astral rays, microtubules or tubulin into consideration. However, the most basic and important key point to understand chromosome movement in anaphase is to grasp exactly and actually the chromosome movement itself in karyokinetic spindles by means of in vivo observations.

With regard to the chromosome movement in anaphase, the following three different types of movements have been discussed confusedly by investigators: 1) cell movement by cilia and flagella, 2) protoplasm streaming, and 3) chromosome movement by kinetochore fibers.

\section{1) Cell movement by cilia and flagella}

In eucaryotes, unicellular plants, protozoa, sperms or zoospores can move freely in unrestricted space by cilia and flagella. Centrioles, one of the structural units of the so-called mitotic apparatus, are cytoplasmic in origin and are primordia for producing locomotive organelles for cell movement. By studying the fine structures of cilia and flagella under the electron microscope, microtubules have been analysed morphologically and the tubulin and dynein biochemically. The results of these new findings have brought important contributions to the interpretation of cell locomotion by cilia and flagella.

The objectives of cell movement in lower organisms may be the provision for the intake of food and the selection of better living circumstances. According to the one gene-one enzyme theory (Beadle and Tatum 1941), it is self-evident that centrioles have nothing to do with the chromosome movement in anaphase.

\section{2) Protoplasm streaming}

This movement is, in general, the movement appearing in cells or plasmodia and covers microscopic distance, but the total distances moved throughout cell life may be immense. As an energy source for protoplasm streaming ATP or tubulin are found in plasmodia, plant and animal cells. Accordingly, the movement of protoplasm is studied as a subject with reference to microtubules, tubulin, ATP, actin or myosin.

Protoplasm streaming is considered to be necessary to maintain the life of cells or of the plasmodia.

3) Chromosome movement in anaphase

This movement is composed of two different behavior patterns viz., the one is 
to form kinetochore fibers and the other to bring chromosomes toward spindle poles.

Before chromosomes begin to move in anaphase, kinetochores of each chromosome are connected with the spindle poles by the formation of kinetochore fibers (chromosomal fibers) and these fibers anchor at both spindle poles (Shimamura 1940). Consequently, both the direction and the distance of chromosome movement in anaphase are set up in advance, and the distance is in general less than 5 micron in both plant and animal cells.

Since the establishment of cytology, the chromosome movement in anaphase had been represented by the 'kinetochore fiber shortening theory' until the introduction of electron microscope studies in cytology. The kinetochore fiber shortening theory has been demonstrated not only in fixation cytology but also in experiments on living mitotic cells under in vivo observations (Wada 1935, Wada and Izutsu 1961, Shimamura 1940). Despite of this fact, it becomes the fashion at present to try to interpret the movement of chromosomes in anaphase by means of microtubules, tubulin or ATP all based alone on the concept of the so-called mitotic apparatus.

If animal cytologists would actually have traced and grasped chromosome behavior in karyokinetic spindles by in vivo observations and also have knowledge of the importance of birefringent figures of mitotic spindles presented by Izutsu et al. (1974), they would not submit to the error of disregarding the kinetochore fiber shortening theory.

4) The chromosome movement in anaphase is one scene of the changes in the mitotic nucleus which continuously take place

The behavior of chromosomes in anaphase is prepared for in advance by continuous changes in structure and function of the mitotic nucleus which take place one after another in teleonomy under gene control.

Apart from the theses on the chromosome (gene DNA) development in the prophase nucleus, a metabolic nucleus, when entering mitosis, alters its outline from sphere to spindle-shap remarkably increasing its volume (Figs. 1a-c) and then becomes a metaphase spindle (Wada 1977). At the end of prometaphase, globular proteins (polypeptides) in the nuclear sap become transformed into fibrous ones which arrange themselves in parallel under the influence of cell polarity. In this way, a prophase nucleus becomes a tactoid as a whole being enveloped with a nuclear membrane the same as it was in prophase (Figs. 1d, e). The birefringent figures of metaphase spindles in living mitotic cells present the changes of intranuclear con-

Fig. 1. Illustrating drawings of the structure and function of a mitotic cell of the Tradescantia staminal hair at the molecular level showing $(\mathrm{a}-\mathrm{g})$ the increase of nuclear volume and also that of the nuclear surface membrane area; (d-e) the unfolding of nuclear sap globular proteins; (d-f) a tactoid formation of fibrous proteins by self-assembling under the influence of cell polarity; (e-f) development of kinetochore fibers and ( $\mathrm{f}-\mathrm{g}$ ) their shortening by ATP (A) convergence at polar regions, and (h) the formation of daughter nuclei by the reappearance of chromatin threads, nuclear sap globular proteins, nuclear membrane and uniform distribution of ATP. Neither the breakdown of the nuclear membrane nor participation of centrioles and asters in spindle fiber formation take place in plant cells nor in animal cells either (i and Fig. 2). The numerals noted in the left upper corner of each cell show the relative value of nuclear membrane surface area (See Table 1). a, early prophase. b, mid-prophase. c, late prophase. d, polar cap stage. e, prometaphase. f, metaphase. $g$, late anaphase. $h$, mid-telophase. $i$, late anaphase of an animal cell. 

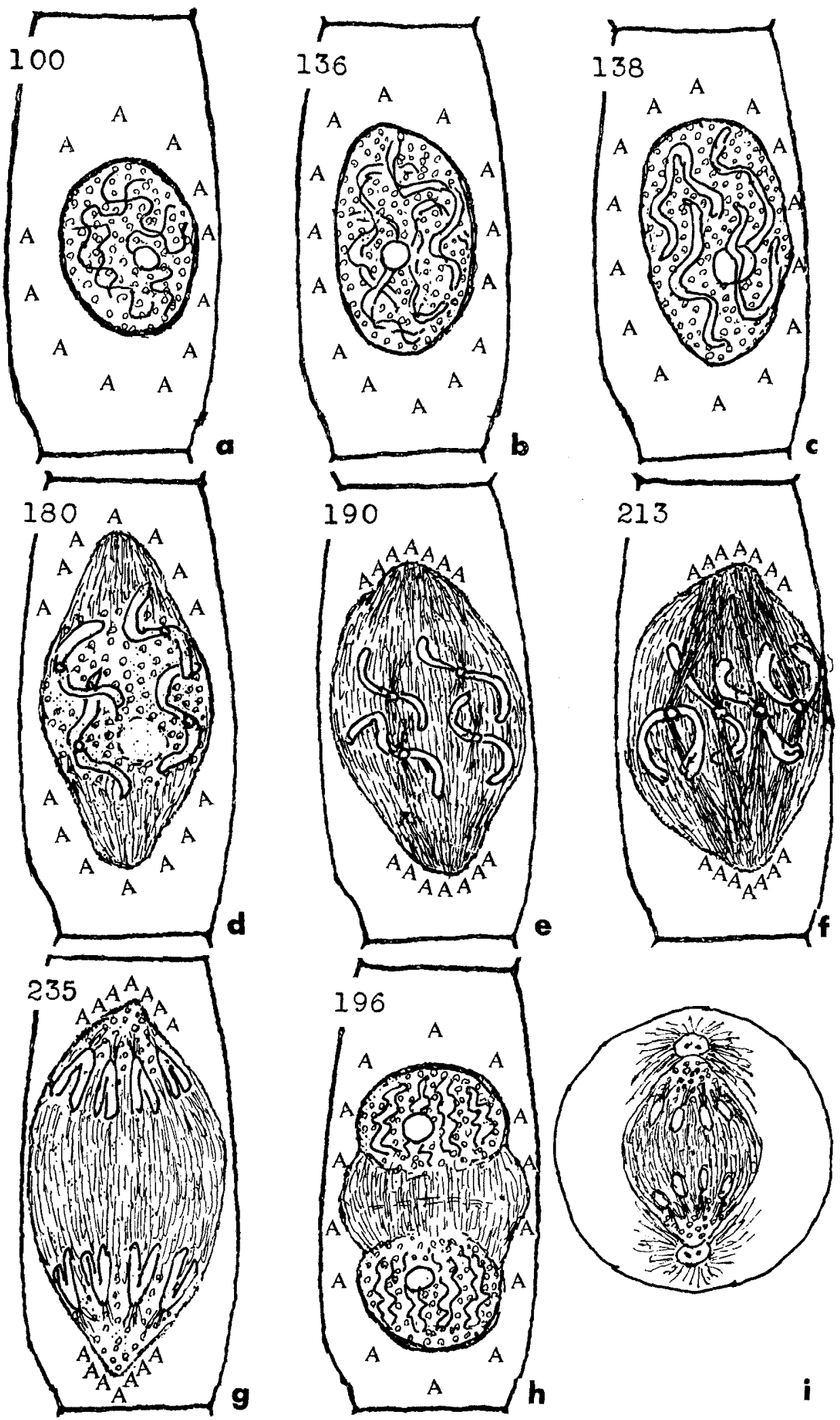
stituents at molecular level (Inoué 1953, Izutsu et al. 1974).

When the formation of the metaphase spindle finishes, the kinetochore substance secreted from kinetochores of each chromosome may physically diffuse into the atractoplasm (parallel arranged spindle back-ground fibrils) and reach the spindle poles. Some of the fibrous proteins of the atractoplasm lying on the path way of the diffusion of the kinetochore substance are bound and become kinetochore fibers (chromosomal fibers) (Figs. le,f). This fiber development in spindles was suggested in the birefringent figures of early metaphase spindles by Izutsu et al. (1974).

As a result of the transformation of the mitotic nucleus from sphere to spindleshape at meta- and anaphase, ATP which uniformly dispersed itself in the cyroplasm of prophase cells (Figs. 1a-c), converges toward both polar regions of the spindle under the influence of the polarized distribution of ions covering the spindle membrane (Wada 1970) (Figs. Id-f). By the same electrophysiological condition, the poleward migration of the centrioles in prometaphase may take place along the surface of the spindle membrane. Consequently, the shortening of kinetochore fibers at the spindle polar regions may be induced by a refolding of the fibrous proteins composing the kinetochore fibers under the activity of ATP at the spindle poles (Wada 1970, 1977) (Fig. 1g).

Based on the continuous changes in structure and function of living mitotic spindles, it is concluded that the karyokinesis is a phenomenon conducted by the nucleus itself (Figs. la-h). There is no room left for centrioles, microtubules, tubulin or actin to participate in the chromosome movement in anaphase even in the mitotic cells of animals (Fig. 1i).

3. Independence of mitotic spindles, their birefringent figures and the genes which control mitosis

In fixation cytology (including electron microscopic studies) very little consideration has been paid 1) to the independence of the karyokinetic spindle enveloped with a biological membrane; 2) to the birefringent figures of mitotic spindles in living cells and their constitutional elements lying opticaly anisotropic at molecular level and 3) to genes which control the structure and function of mitotic spindles.

1) Mitotic spindles in living cells observed under dark-field microscope

By in vivo observations on the meiosis of pollen mother cells of Tradescantia under the dark-field microscope, Fujii (1931) confirmed that the ground substance occupying the spindle area is in a state of an optical vacuum surrounded with immense numbers of granules in various sizes illuminated in the cytoplasm. Fujii proposed a name 'atractoplasm' (spindle protoplasm) for the spindle ground substance appearing as an optical vacuum and suggested that the atractoplasm may originate from the nuclear sap. It is natural to conclude that the dark space, as a physical phenomenon, where no moving granules can enter, is enveloped by a biological membrane.

In fixation cytology, however, the same spindle area surrounded with granules is conceived of as appearing due to the breakdown of the nuclear membrane before 
spindle formation. Based on this misinterpretation, the following assumptions have become prevalent: 1) in animal cells, centrioles and astral rays participate in the formation of spindle fibers and 2) in plant cells, by the mixing of kinoplasm (particular cytoplasmic zone surrounding the prophase nucleus) with karyoplasm, the spindle fibers are produced. These hypotheses, at present, are modified in a certain degree. The misinterpretation of the breakdown of the nuclear membrane before spindle formation is still described in cytology books and precludes the elucidation of the mitosis mechanism from reports introducing various new findings in biology and genetics.

2) Mitotic spindles in living cells observed under the polarization microscope Inoué (1953) has succeeded in observing mitotic spindles in living cells of plants and animals under the polarization microscope. The birefringent figures of mitotic spindles have been revealed to correspond to the optical vacuum area appearing under the dark-field microscope (Fujii 1931). Based on the observations mentioned above, it may be concluded that the atractoplasm is constituted of submicroscopic fibrils which arrange themselves optically anisotropic lying parallel to the spindle axis (Wada 1950, 1966). In this way Inoué has for the first time shed light on the elucidation of the spindle mechanism at the molecular level.

Based on Fujii's atractoplasm theory and Inoué's birefringent figures of mitotic spindles in living plant and animal cells, the present author has proposed with regard to the developmental process of karyokinetic spindles the following working hypothesis (Wada 1966). With the optical anisotropic arrangement of molecules in karyokinetic spindles, one cannot then consider the exception of the transformation (unfolding) of nuclear sap proteins (polypeptides) into fibrous ones which would arrange themselves in parallel under the influence of cell polarity. Furthermore, it is assumed that the transformation of nuclear sap proteins may be induced by the catalyzer-like reaction of nucleolar RNA in prometaphase (Wada 1977).

3) The genes which control the formation and disintegration of the karyokinetic spindle

Based on in vivo observations and birefringent figures of living mitotic spindles, the author has reached the conclusion that the karyokinetic spindle is a transformed metabolic nucleus itself $(1950,1966,1977)$, and has suggested that it is the genes which may control the appearance of the karyokinetic spindle. The author calls the gene(s) a 'spindle formation inducer gene' (SFI) collectively and for convenience. With regard to the behavior of spindles in telophase, the author (1970) has named the gene(s) which may repress the structure and function of spindles a 'spindle formation repressor gene' (SFR).

The activity of the SFI gene is the starting point of cell proliferation and that of ontogenesis. The activity of the SFR gene is to repress mitosis and initiate the starting point of cell differentiation. Accordingly, the appearance of cell proliferation and that of cell differentiation take place antagonistically. This relationship is a very important and basic principle in eucaryotes but is completely overlooked in fixation cytology. 
So long as the SFI gene and the SFR gene are active in a balanced state, individuals of all the eucaryotes can produce normal tissues and organs and continue growth and maintain their species. By reason of internal or external damage to the gene DNA, should a deficiency occur in the activity of the SFR gene, the organism would begin to suffer from unrestricted cell proliferation without cell differentiation (Wada 1979). Based on this viewpoint and considering the principle of karyokinesis, the author proposes the opinion that the ultimate cause of carcinogenesis is unitary, namely the deficiency of the SFR gene (Wada 1974, 1979).

4. Comparing electron microscopic figures (EMFs) of mitotic spindles with their birefringent figures, the former are of no usefulness for the study of the structure and function of mitotic spindles

At present it has become the fashion in cytology to study cells with the electron microscope (EM). Even though the EM has excellent resolving power superior to any light microscope, the electron microscopic figures (EMFs) of mitotic spindles practically fail to bring out the submicroscopic structures of the mitotic spindles which are well known as the continuous fibers in fixation cytology, and also fail to bring out an optical anisotropic arrangement of spindle back-ground fibrils observed as birefringent figures in living mitotic cells under polarization microscopes.

According to Forer's estimation the microtubules (MTs) comprised in spindles occasionally make up nearly 5-10 per cent of the spindle volume (cited in Bajer's 'Zipper hypothesis' 1975). Such EMFs apparently reveal that 90-95 per cent of spindle back-ground fibrils disappear as artifacts due to inadequate fixatives. Different from spindle back-ground fibrils (continuous fibers in classical cytology), kinetochore fibers protected with a kinetochore substance are considered to be resistant to liquefaction induced by glutaraldehyde-containing fixatives and to remain in a swollen state, as if they were composed of MTs.

1) To select fixatives corresponding to the hydrogen ion concentration of each organelle is a basic principle in fixation cytology

The reason for the disappearance of more than 90 per cent of spindle background fibrils in EMFs is very clear.

Formerly by Naylor (1926) and recently by Yasui and Fujii (1951), it has been concluded that the least artifact effect and the best staining effect for nuclear contents (chromatin threads, chromosomes, nucoleli, etc.) are obtained by fixatives with $\mathrm{pH} 5.6-3.5$ and for organelles in cytoplasm (plastids, mitochondria, centrioles, astral rays, etc.) by fixatives with $\mathrm{pH}$ 5.6-7.8. From detailed investigations on fixation and staining effects of the nuclear contents in metabolic as well as mitotic states, Yasui and Fujii (1951) have reached the conclusion that the least artifact effect and the best staining effect of mitotic spindles are obtained with fixatives in the narrow range of $\mathrm{pH}$ value 5 .

With the treatment of strong acidic fixatives, such as acetocarmine, mitotic spindles completely disappear with the liquefaction of all kinds of spindle fibers 
leaving chromosomes intact. Similarly in treatment with alkaline fixatives or weak acidic ones, all of the spindle background fibrils (continuous fibers in classical cytology) completely disappear but swollen kinetochore fibers and the outline of spindle are vaguely seen as already mentioned above. These artifacts practically appear in the images of mitotic spindles treated with glutaraldehyde-containing fixatives which are always on the alkaline side far apart from $\mathrm{pH} 5.0$.

2) No microtubules (MTs) exist in the karyokinetic spindles in both plant and animal cells

It may be the unscientific practice that fixatives are selected according to the types of microscopes but not according to the differences of $\mathrm{pH}$ values of the organelles to be studied.

Fixatives containing glutaraldehydes were for the first time applied to the study of enzyme histochemistry under the electron microscope by Sabatini et al. (1963). Due to the alkaline nature of fixatives, glutaraldehyde-containing fixatives have been used widely and have contributed to studies on mitochondria, chloroplasts and many organelles or inclusions and structures in cytoplasm, e.g. endoplasmic reticulum, centrioles, astral rays, cilia and flagella. The success in the elucidation of cell movement by MTs morphologically and by tubulin biochemically has brought on the fashion of interpret into the chromosome movement in anaphase by MTs and tubulin also. However, such interpretations are due to a complete miscalculation as stated in the foregoing chapter (See p. 601). It is unfortunate that the swollen state of kinetochore fibers treated with glutaraldehyde-containing fixatives (the least serviceable $\mathrm{pH}$ condition as a fixative for karyokinetic spindles) apparently, resemble microtubules obtained from cilia, flagella, or astral rays fixed with glutaraldehyde-containing fixatives (the most serviceable $\mathrm{pH}$ condition as a fixative for the fine structures of cytoplasmic origin).

In living mitotic cells in both plants and animals, neither the cause and effect relation nor the precursors for producing MTs are present in prophase nuclei before and after their transformation into karyokinetic spindles.

3) EMFs which correspond to the birefringent figures of spindles in living cells

Using metal salt solutions $\left(\mathrm{CdCl}_{2}, \mathrm{MnSO}_{4}, \mathrm{~Pb}\left(\mathrm{NO}_{3}\right)_{2}, \mathrm{Cu}\right.$-acetate, etc.) as the observation medium, the author was for the first to succeed in visualizing the presence of the spindle membrane in meiosis of pollen mother cells of Tradescantia and Hosta and also in meiosis of the spermatocytes of Atractomorpha bedeli (Wada and Fukunaga 1957) and also by application of metal salt solutions to fixatives, the presence of spindle membrane, back-ground fibrils of spindles, and constitutional fibrils of the kinetochore fibers were demonstrated in fixed preparations of mitotic cells in root tips in Vicia faba and Allium cepa (Wada et al. 1963), in pollen mother cells of Tradescantia reflexa and Zephyranthes candida (Wada and Kusunoki 1964a), in mitosis of Ehrlich ascites tumor cells (Wada and Kusunoki 1964b), in mitosis of Hemicentrotus pulcherrimus (Wada et al. 1964), in spermatocytes meiosis of Chloealitis geniculaributs (Fig. 2), and Bombyx mori (Nakao, Nakanishi and Wada 1968).

Satô $(1958,1959,1960)$ fixed the pollen mother cells of Lilium longiflorum 
with $1 / 20 \mathrm{M} \mathrm{CdCl}_{2}$ solution and ethanol and succeeded in visualizing spindle background fibrils, kinetochore fibers, their constitutional fibrillar elements and cell plate composed of fibrils arranged in double layers. All of these images corresponded to the birefringent figures demonstrated in living mitotic cells of plants and animals (Inoué 1953, Izutsu et al. 1974).
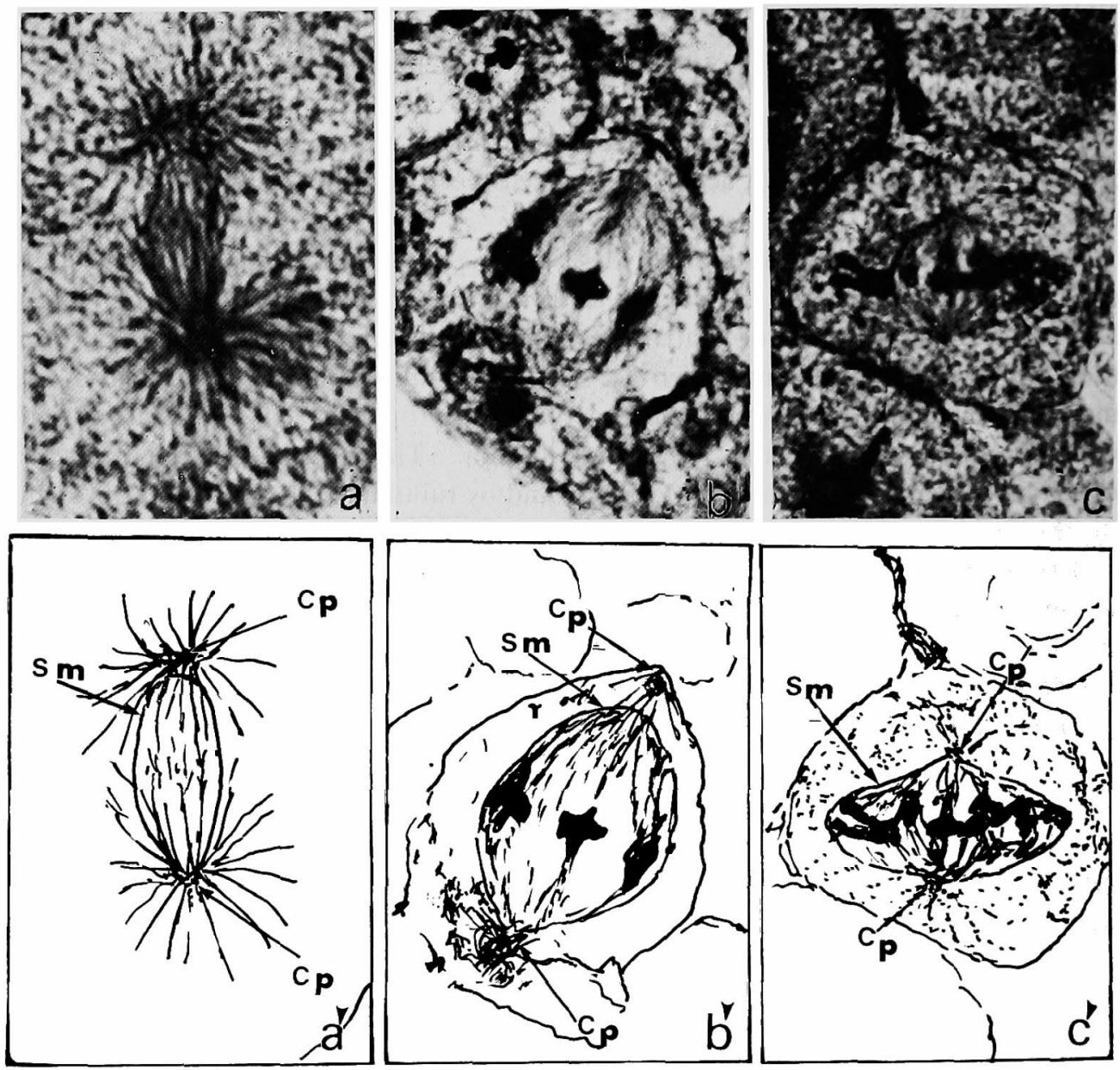

Fig. 2. The independence of karyokinetic spindles enveloped with a nuclear membrane (spindle membrane) from the cytoplasm was demonstrated by an improved fixation technique. a, a metaphase spindle in a sea urchin egg cell, Hemicentrotus pulcherrimus (Wada et al. 1964). b-c, metaphase spindles of spermatocyte meiosis in the grasshopper, Chloealitis geniculaributs. b, metaphase spindle in a first meiosis. c, metaphase spindle in a second meiosis (Nakao et al. 1968). The cells in a-c were pre-treated with $1 / 10 \mathrm{M} \mathrm{MnSO}_{4}$ solution and fixed with Bouin's fluid. $2 \mathrm{a}^{\prime}-\mathrm{c}^{\prime}$. Illustrative drawings of photographs of 2a-c. Centrioles adsorp the outside of the spindle membrane at spindle poles $\left(a^{\prime}\right.$ and $\left.c^{\prime}\right)$ and the inside of the plasm membrane $\left(b^{\prime}\right)$ showing no relation to the spindle fiber formation. These are only one instance among many that the spindle of eucaryotic cells enveloped with a nuclear membrane is always independent of the cytoplasm. The breakdown of the nuclear membrane before spindle formation is a great mistake due to classical fixation techniques (including glutaraldehyde containing fixatives). The spindle axis in Fig. 2a appears slightly declined. Cp: position of centrioles, $\mathrm{Sm}$ : spindle membrane (nuclear membrane in meta- and anaphase stages). 

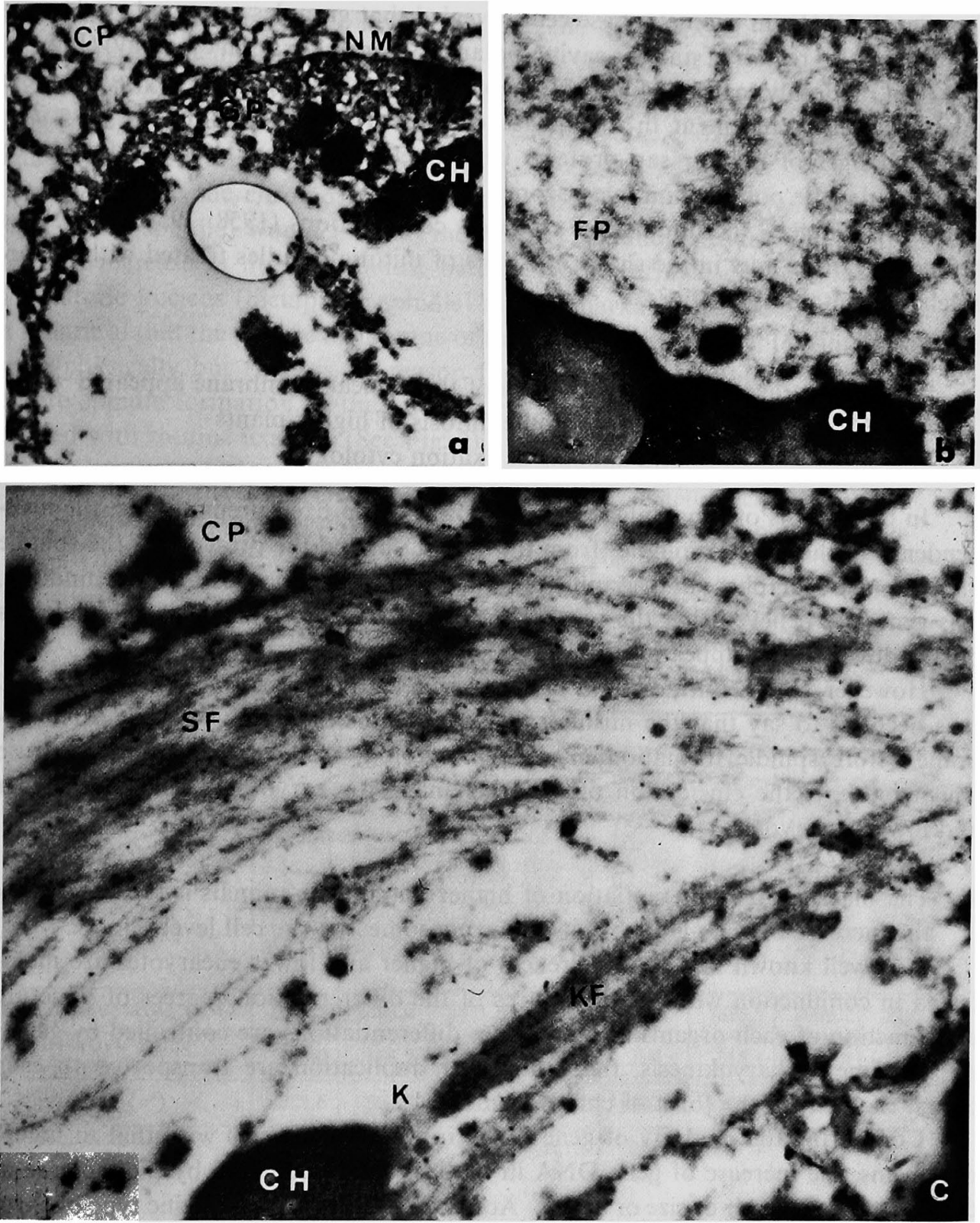

Fig. 3. Electron micrographs of the development of spindle background fibrils and the fine structure of the kinetochore fiber in a pollen mother cell of Lilium longiflorum fixed with $1 / 20 \mathrm{M} \mathrm{CdCl}_{2}$ solution and ethanol (Satô 1960). a, assembling of nuclear sap globular proteins (precursors of spindle background fibrils) (GP) at a polar region inside of a nuclear membrane (NM) (a polar cap stage). $\times 3,000$. b, unfolded fibrous proteins $(\mathrm{FP})$ appear but do not show yet definite orientation. Chromosomes $(\mathrm{CH})$ crowded into a mass. $\times 6,000$. c, due to a parallel arrangement of each fibril (SF) under influence of cell polarity, a prometaphase nucleus forms a tactoid and becomes spindle-shape (a metaphase spindle) maintaining its independency of the cytoplasm (CP) composed of randomly scattered amorphous glanules. A kinetochore fiber (KF) developed from a kinetochore $(\mathrm{K})$ reveals it to be composed of a bundle of spindle background fibrils. $\times 7,000$. (Courtesy of Dr. S. Satô). Satô's EMFs attribute the correspondence to the birefringent figures of karyokinetic spindles as demonstrated in living mitotic cells by Inoué (1953) and Izutsu et al. (1974) under polarization microscope. No microtubules exist inside the metaphase spindle. The demonstration of the spindle membrane should be dependent on other techniques such as used in Fig. 2 or Fig. 4. 
Satô's EMFs in prometaphase cells revealed that granules appeared converging toward a polar region of nuclear cavity (polar cap stage in fixation cytology) (Fig. 3a) and then randomly scattered fibrils fully appeared in the nuclear cavity, just before their parallel arrangement in metaphase of the formation of a tactoid (Fig. 3b). These changes of nuclear sap proteins in prometaphase would not be able to be traced under the polarization microscope.

Unfortunately, the importance of Satô's contributions $(1958,1959,1960)$ have been disregarded now in the shade of EMFs of mitotic spindles treated with glutaraldehyde-containing fixatives.

5. The reason for the breakdown of the nuclear membrane appeared with restrictions in the mitosis of higher plants and animals in fixation cytology

In the mitosis of fungi, algae or protozoa, even in fixed preparations, the independence of the mitotic spindles from the cytoplasm and the presence of the spindle membrane in metaphase and anaphase are well known facts. Despite these findings, it is generally believed that the nuclear membrane in higher plant and animal cells breaks down and disappears before spindle formation.

However, this contradiction has never been discussed in cytology books. It is needless to say that the misinterpretation on the breakdown of nuclear membrane before spindle formation in classical cytology is the most important cause which prevents the elucidation of the mechanism for karyokinesis in both higher and lower eucaryotes.

1) The macroscopic differentiation of higher plants and animals is represented by the increase of gene DNA (that of chromosomes) at the cell level

It is well known that the differences of higher and lower eucaryotes are manifested in conjunction with the differences of the differentiation degrees of structure and function of each organism; all of these differentiations are controlled by genes. At the time of karyokinesis, the genes after duplication are transported to each daughter nuclei in the form of chromosomes.

Comparing the quantity of gene DNA in lower organisms with that in higher organisms, the increase of gene DNA in the latter is represented by the increase of chromosomes number or size or both. Accordingly, the species-specific type of chromosomes (karyotypes) are important subjects of static morphology in fixation cytology. However, elucidations on various aspect in the dynamic morphology of chromosomes such as the duplication of gene DNA or development of chromatin threads into chromosomes or chromosome movement in anaphase would not be achieved from dependence on fixation cytology alone.

2) The increase of nuclear volume and the strained state of the spindle membrane in higher plant and animal cells

The ultimate purpose of karyokinesis is to divide and to transport the gene DNA of the mother cell equally into daughter nuclei in quality and in quantity. 
In the first step to carrying out this purpose, the kinetochore of each chromosome must arrange themselves in one plane at the equator of the metaphase spindle (Wada 1966). In the second step, the distance between both spindle poles must be long enough to separate the split daughter chromosome ends completely, after each kinetochore has reached its respective pole.

To achieve the chromosome movement mentioned above, metaphase nucleus (metaphase spindle) would have to increase its volume considerably. As a result of such increase in nuclear volume before spindle formation, the surface area of the mitotic nucleus (metaphase spindle) must remarkably increase. Consequently, it is natural that the surface membrane of the metaphase spindle enters into a strained state physically but there is no reason for the nuclear membrane to break down before spindle formation, if the mitotic cells in higher plants and animals are not treated with routine fixatives (See Fig. 2).

Table 1. The change in surface area and volume of a mitotic nucleus in a living Tradescantia staminal hair cell calculated according to the formula of the ellipsoid of rotation on the outline of the nucleus in each stage $(a-h)$. a, early prophase

$(14: 00) . \quad b$, mid-prophase $(14: 35)$. c, late prophase $(15: 35)$. d, polar cap stage $(16: 20)$. e, prometaphase $(16: 25)$. f, metaphase $(16: 45)$. $g$, late anaphase $(17: 03)$. $h$, mid-telophase $(17: 15)$. The numerals in parentheses indicate the time of observation (Wada 1950)

\begin{tabular}{|c|c|c|c|c|c|c|c|c|}
\hline & a & $b$ & $c$ & $d$ & e & $f$ & $g$ & h \\
\hline & & & & & & & & \\
\hline Surface area $\left(\mu^{2}\right)$ & 1685.9 & 2296.2 & 2331.6 & 3041.4 & 3321.2 & 3592.7 & 3960.2 & 3297.6 \\
\hline $\begin{array}{l}\text { Relative value } \\
\text { of surface area }\end{array}$ & 100.0 & 136.2 & 138.3 & 180.4 & 197.0 & 213.1 & 234.9 & 195.6 \\
\hline Volume $\left(\mu^{3}\right)$ & 6333.5 & 9500.3 & 10038.6 & 14035.0 & 16967.4 & 19488.2 & 21818.9 & 15979.4 \\
\hline $\begin{array}{l}\text { Relative value } \\
\text { of volume }\end{array}$ & 100.0 & 150.0 & 158.5 & 221.6 & 267.9 & 307.7 & 344.5 & 252 \\
\hline
\end{tabular}

On the contrary however, in lower organisms such as algae, fungi or protozoa, it is not necessary to increase the volume of their mitotic nuclei (metaphase spindles), because their chromosomes are few in number and small in size. In other words, mitotic nuclei in lower organisms remain constant in volume, in their surface area, and in their physical state. Consequently, their spindle membrane remains constantly the same as their prophase nuclear membrane does, when fixed.

Cytologists know but vaguely of the increase of nuclear volume during prophase but practically there is no procedure to confirm the increase of nuclear volume during mitosis in one and the same cell in fixation cytology.

In in vivo observations of Tradescantia hair cells, the author (1950) continuously 
photographed the mitotic nucleus from the beginning of prophase to mid-telophase by time lapes method in one and the same cell and calculated the volume and surface area of the nucleus in each stage according to the formula of the ellipsoid of rotation (Wada 1950). The result of this calculation revealed that the nucleus at metaphase (metaphase spindle) increased about three times in volume and about 2 times in surface area more than those in metabolic nucleus (Table 1).

From the result of this calculation, it is concluded that the nuclear membrane enters into a strained state physically, when the metabolic nucleus developes into a metaphase spindle. Consequently, it is natural that the strained spindle membrane may break down into pieces at the moment of coagulation due to contact with fixatives (including glutaraldehyde-containing fixatives). Thus, no spindle membrane in higher plants and animals can ever be found in fixed preparations as a resultant of artifact.

On the contrary, in lower plants and animals, their nuclei can become karyokinetic spindles without increasing their nuclear volume, so consequently the physical state of their spindle membrane is constantly the same as it was in prophase. Thus, no breakdown of the nuclear membrane when fixed takes place before spindle formation.

3) EMFs which demonstrate the strained state of the spindle membrane

It is generally difficult to determine the physical state of any membrane which envelopes intracellular organelles directly or indirectly. Fortunately, however, the strained state of the spindle membrane can be demonstrated by means of electron microscopy.

To avoid the breakdown of the nuclear membrane before spindle formation by routine fixatives (including glutaraldehyde-containing fixatives), Sprey and Hasche (1972) studied the ultrastructural changes of the nuclear membrane in the mitotic cells of Lepidium sativum $\mathrm{L}$. by means of the freeze fracture and etching technique. They observed the nuclear membrane and its membrane pores not only in interphase but also in prophase nuclei as well as meta-, ana- and telophase spindles. They counted the number of pores per 1 unit area $\left(\mu^{2}\right)$ of nuclear membrane from interphase to telophase and found that the number of membrane pores in interphase nuclei was 20 (per $\left.\mu^{2}\right)$ in average, while the pores in metaphase spindles were 5-15 (per $\mu^{2}$ ) (Fig. 4).

The decrease of membrane pores per unit area indicates the increase of membrane surface area of metaphase spindles as a result of the increase of nuclear volume during prophase. Consequently, it is natural to conclude that the nuclear membrane in meta- and anaphase enters into a strained state physically.

The freeze etching images of mitotic spindles presented by Sprey and Hasche (1972) revealed an important evidence that the breakdown of the nuclear membrane before spindle formation in higher plant and animal cells is nothing but an artifact induced by routine fixatives (including glutaraldehyde-containing fixatives).

4) Karyokinesis functions according to a common principle in plant and animal cells 
In order to elucidate any complex phenomena such as the mechanism of mitosis, it is scientific common sense to select a material with the simplest organization. Because of this consideration, plant cells in mitosis are apparently superior to animal cells since the latter contain centrioles and astral rays in the cytoplasm. Among animal cells, sea urchin cells are most inadequate to study mitosis, because their
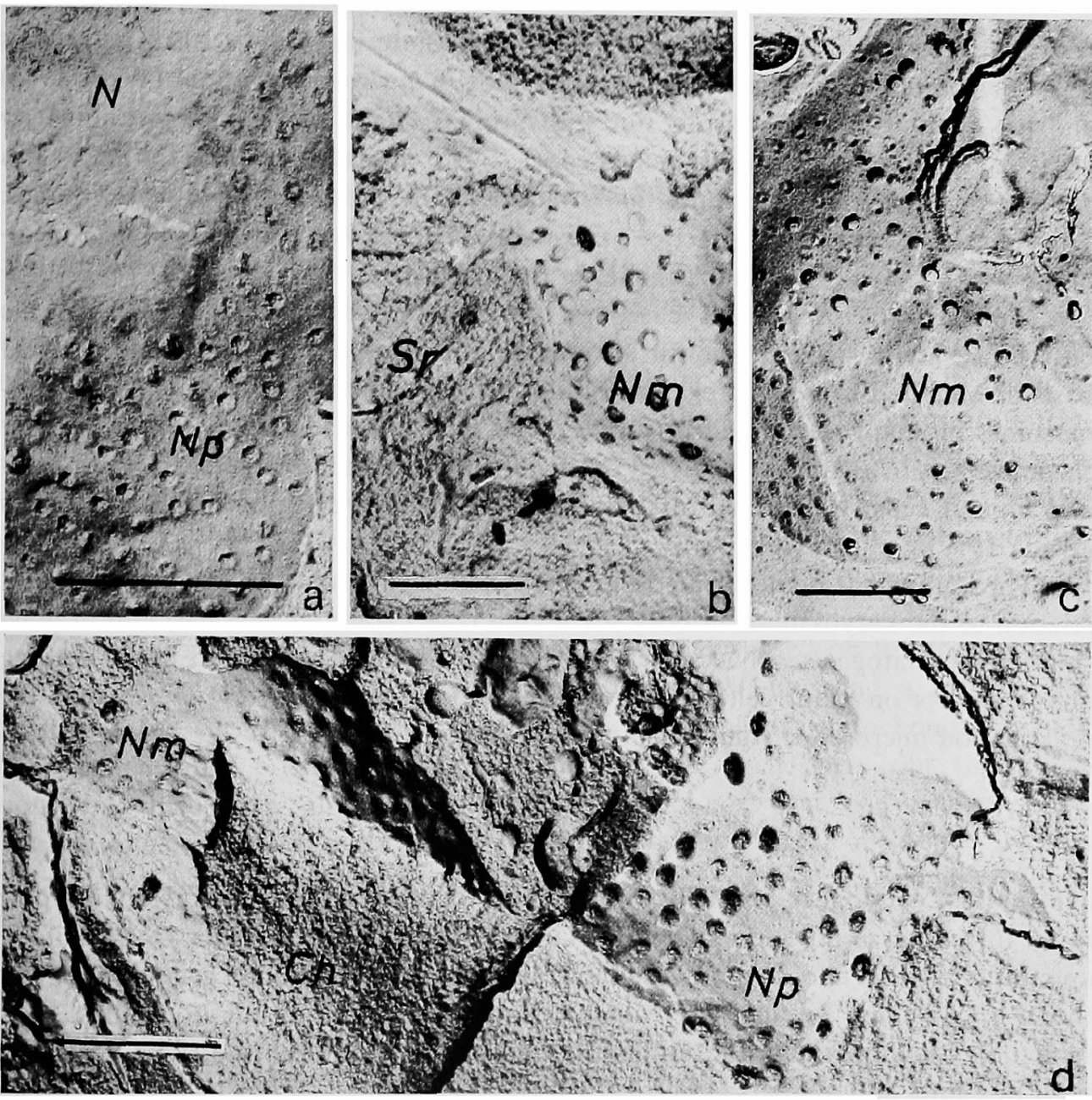

Fig. 4. Electron micrographs of the nuclear envelope (spindle membrane) in the mitotic cells of Lepidium sativum $\mathbf{L}$. demonstrated by means of freeze fracture and etching technique (Sprey and Hasche 1972). a, a mid-prophase nucleus $(N)$ enveloped with a nuclear membrane and membrane pores $(N p)$. b, nuclear membrane in a meta-anaphase cell. Nuclear envelope $(\mathrm{Nm})$ appears covering the spindle region $(\mathrm{Sr}) . \quad \mathrm{c}$, nuclear membrane $(\mathrm{Nm})$ in an early telophase cell. d, in a late anaphase cell, a part of the nuclear envelope $(\mathrm{Nm})$ with membrane pores $(\mathrm{Np})$ appears touching chromosome $(\mathrm{Ch})$. (Courtesy of Dr. B. Sprey). These EMFs offer the following two very important items of evidence. One is that the karyokinetic spindle covered with a nuclear envelope (spindle membrane) is morphologically independent of the cytoplasm, and the second is that the spindle membrane is physically in a strained state judging from the dispersed distribution state of membrane pores of the nuclear envelope which covers the enlarged volume of the metaphase spindle. Scale: $1 \mu \mathrm{m}$. 
mitotic spindles are covered with particularly developed astral rays from centrioles at both spindle poles, and the behavior of the chromosomes in the anaphase spindles are practically impossible for tracing chromosome movement in the in vivo observations as well as in fixed preparations (Wada 1976). The use of sea urchin cells by animal cytologists is nothing but a long-established custom in classical cytology and is not one dictated by requirements of modern biological sciences.

In contrast to animal cells, the plant cells in cormophytes which have no centrioles always reveal mitotic spindles in a naked state in cytoplasm. The mitosis of Tradescantia staminal hair cells is easy to study in in vivo observations (Wada 1943) and the observations are carried out with ordinary light microscopes without any necessity to use phase contrast microscopes. The optical system of the latter has a structural deficiency in making hallows inevitablly around optical dense bodies. This defect encourages the practice of disregarding the presence of biological membrane which envelopes each organelles in the cytoplasm.

Mendel's law of genetics based on the results of hybridization experiments on the pea are at present true for all plants, animals and mankind. The physical basis of Mendle's low of segregation is due to the disjunction process of sister chromosomes in anaphase of meiosis in peas. In other words, the separation of sister chromosomes in anaphase is carried out by one and the same principle in both plant and animal cells, irrespective of plants having neither centrioles nor astral rays (Wada 1977).

The author has demonstrated that the centrioles in animal cells have no direct relation to the karyokinesis in both sea urchin cells (Wada et al. 1964) and in grasshopper spermatogenesis (Nakao et al. 1968) (Fig. 2). The author has heard nothing about reports on plant cell mitoses investigated by animal cytologists except the polarization microscope studies by Inoué (1953).

\section{Centrioles}

Animal cytologists believe that centrioles together with astral rays participate in the formation of mitotic spindles and label these organelles together as the 'mitotic apparatus' (Mazia and Dan 1952). However, standing on the one gene-one enzyme theory (Beadle and Tatum 1941) which becomes applicable to all organelles, their structures and functions, the author has discussed the raison d'être of centrioles both in plants and animals $(1969,1970,1972,1977)$ and will repeat here the irrelevance of centrioles to mitosis.

1) Centrioles as useless organelles in the course of evolution in eucaryotes disappeared from higher plant cells

In the case of individual cell movement in eucaryotes (e.g. unicellular plants and protozoa), the centrioles play the role of primordia for locomotive organs (viz. cilia and flagella). Looking upon centrioles in a broad sense, the author holds the opinion as already stated (Wada 1977) that all of the cells endowed with movability possess centriole(s) irrespective of having differentiated locomotive organelle(s) such as cilia or flagella. Animal cells also even those in tissues exert movement 
for taking in nutriments outside of cells. In the case of plant cells, these latter can effect nutrition within themselves by photosynthesis. Accordingly, plant bodies are constituted with collectively bound tissue cells fully able to utilize solar energy for photosynthesis. Consequently, cells in higher plants are no longer necessity to retain either movability or centriole(s). Thus the centriole disappears from plant cells as a useless organelle in the course of evolution (Wada 1972).

It is natural and clear that centrioles have no relationship to the mechanism of karyokinesis in both plant and animal cells.

2) Criticism of the so-called mitotic apparatus based on the one gene-one enzyme theory

The mitotic apparatus which is commonly referred to by animal cytologists is composed of centrioles, astral rays and the mitotic spindle. The raison d'être for centrioles in both plant and animal cells has been clarified in the foregoing sentences.

The appearance of astral rays shows remarkable differences among different kinds of animals, and are considered to appear secondarily due to an interaction between a centriole-bound substance and cytoplasm (Wada 1972). On the contrary, the mitotic spindles occupying the center of the mitotic apparatus are intrinsically independent organelles transformed from metabolic nuclei which participate in the mechanism of karyokinesis. The formation of cilia and flagella as well as that of microtubules and tubulin are controlled by genes specific for centrioles. This interpretation is at present but scientific common sense in accordance with the one gene-one enzyme theory (Beadle and Tatum 1941).

The concept of the mitotic apparatus is ultimately based on fixation cytology and it is rather an artificial approach to postulate a staging and a prop in the cytoplasm for carrying out nuclear division. Chloroplasts, mitochondria, many other intracellular organelles and even the centriole itself carry out their divisions by themselves automatically under control of organelle-specific genes. The metaphase spindle itself is a transformed feature of the metabolic nucleus to carry out karyokinesis (equivalent division of gene DNA) in teleonomy. It is wondered at that animal cytologists seem to have forgotten the wise saying of Virchow (1855) and Flemming (1882) "Omnis cellula e cellula" and "Omnis nucleus e nucleo".

According to the present advances in the genetics of molecular architecture, the meaning of the one gene-one enzyme theory (Beadle and Tatum 1941) should be enlarged to indicate that one gene controls one unit architectural molecular assembly. Consequently, the appearance and disappearance of spindle fibers at the nuclear division of eucaryotes is a typical example of a self-assembling reaction (Communication of Iino 1980).

3) Biological significance of isolated spindles from the so-called mitotic apparatus At present some animal cytologists make displays of the isolation of spindles away from the mitotic apparatus by biochemical procedures carried out at $\mathrm{pH} 6.2$ in most cases so far reported (Sakai et al. 1979). These isolated spindles were investigated under the light microscope, phase contrast microscope and polarization microscope. 
Strictly speaking, the centrioles and a greater part of the astral rays covering the spindle body are removed from the mitotic apparatus biochemically. However, there is no evidence that the astral rays physically adsorped on the surface membrane of spindles were completely removed from the membrane. It may be technically impossible to isolate adsorped microtubules, from the spindle surface without destroying the spindle membrane itself. To confirm the presence of microtubules and tubulin in the mitotic spindles, animal cytologists should demonstrate the presence of microtubules and tubulin or their precursors in the prophase nucleus but it may be a demonstration in vain.

In the living state of mitotic spindles, the spindle membrane is extremely delicate and fragile and in a strained state physically. The spindles cannot stand up under any routine biochemical and biophysical treatments designed to isolate them from the cytoplasm. To elucidate the structure and function of spindles molecular-biologically, in future investigations will be essential and necessary to develope new and creative techniques for isolating mitotic spindles from cytoplasm (Wada 1979).

7. Biological significance of the differences in cytokinesis between plant and animal cells

This subject is not always a problem in the elucidation of karyokinesis but yet a very important one which has been disregarded in fixation cytology just as the antagonistic appearance of cell proliferation and cell differentiation have been overlooked (Wada 1979).

The cytokinesis which succeedingly occurs after the karyokinesis is the starting point of ontogenesis. To maintain individual life, each eucaroyte must carry out the intake of nutriments. For this biological viewpoint, it is natural that the cytokinesis of plant cells be performed differently from that of animal cells: the former is furnished to conduct the autotrophic mode of life and the latter the heterotrophic mode of life in teleonomy.

1) Cytokinesis in plant cells makes a collective mass of bound cells for photosynthesis

In early telophase, anaphase spindles in plant cells alter into phragmoplasts by hydration (Wada 1935, 1966, Olha and Bozzola 1972). On the equator of the phragmoplast, a cell plate appears centrifugally and the cell wall develops centripetally from where the cell plate reaches the mother cell wall (Wada 1966). Consequently, the newly appearing two daughter cells are bound together with a cell wall. Thus, plant cells proliferate and make a collective mass of bound cells. Therefore, they have no further necessity to maintain either centrioles or cell movability.

The morphogenesis of the higher plants equipped with a cell wall in each cell is advanced by the differentiation of roots, stems, branches and leaves for carrying out photosynthesis effectively.

2) Cytokinesis of animal cells makes a collective mass of movable cells for the intake of nutriments 
In telophase of animal cells, spindles remain for a while as spindle residues in mother cells, but the cells are separated into two daughter cells by the formation of cleavage at the equator of the mother cell. The each daughter nucleus and also the centrioles lying at each pole of the spindle residues begin to occupy the center of the daughter cells which maintain their movability by the presence of the centrioles.

In the case of animal cells, the daughter cells are independent of each other and movable. These cells compose of a collective mass maintaining their independency and movability. In the morphogenesis of animals, cells differentiate into tissues and organs, and each individual is equipped with organs for digestion, locomotion, sensation, reproduction and also with many other organs.

In protozoa or in unicellular organisms, the locomotive organs are represented by cilia and flagella which develop from the centriole. In higher animals or multicellular organisms, locomotive organs differentiate into legs, wings, or arms. Taking all these locomotive organs in lower and higher animals into consideration, the author (Wada 1978) has presented an assumption that the centriole is a cardinal organelle in the cytoplasm which may regulate the locomotion of the cell itself as well as the formation of the locomotive organelle in teleonomy under gene control.

The centriole is intrinsically indifferent to the movement of chromosomes in anaphase but exists in somatic as well as in sexual cells in the animal kingdom, because animal cells are heterotrophic and require movability for the intake of nutriment from other cells.

8. Insoluble problems in mitosis

With regard to insoluble problems in mitosis, there are two categories of phenomena: one of these will be analysed and elucidated in the future by present chemical or physical procedures, but the other phenomenon is manifested in the vitality incorporated in each individual and it is difficult to treat these phenomena separately and physically.

1) The mitotic phenomenon which will be elucidated in the future by present physical and chemical principles

Although the existence of the spindle membrane seems to be disregarded by animal cytologists, it seems to be common sense in modern biology that any organelles in living cells are enveloped with a biological membrane. Therefore, the presence of the spindle membrane itself is no longer a problem to be discussed in this chapter.

i) The quantitative analysis of the transformation of nuclear sap globular proteins into fibrous ones: Taking into account the birefringent figures of Inoué (1953) and Izutsu et al. (1974) together with Fujii's atractoplasm theory (1931), these birefringent figures of the spindle have led the author to the conclusion that a reversible folding $\rightleftarrows$ unfolding of nuclear sap proteins (polypeptides) must have occurred without participating centrioles and the astral rays in spindle fiber formation.

In the field of molecular biology, the folding and unfolding of polypeptides is demonstrated in vitro quantitatively by means of dichroic micro spectroscopy 
(A. Wada 1972). The technical development and application of this technique to in vivo observations will be improved in the future, and the reversible transformation of nuclear sap proteins in the formation and disappearance of spindles will be elucidated quantitatively at the molecular level (Wada 1977).

ii) The change of ATP distributions in the mitotic cell before and after the appearance of the metaphase spindle: The demonstration of ATP convergence around the spindle poles may not be a difficult problem in cytochemistry. However, by a misunderstanding about the presence of the nuclear membrane (an intracellular biological membrane) which envelops the metaphase spindle, the demonstration of the ATP distribution change in mitotic cells has been disregarded for a long time. If the convergence of ATP at the anaphase spindle poles were to be confirmed, the disintegration or the shortening process of kinetochore fibers in the polar region of the anaphase spindle would also be elucidated biochemically as a subsequent step.

iii) The structure and function of the spindle membrane at molecular level: Different as it is from the intercellular cell membrane, the author has scarcely known in detail at the molecular level the structure and function of the intracellular biological membrane. However, the subject of the intracellular biological membrane both in the metabolic as well as in the mitotic state is essential to clarify the mechanism of mitosis at the molecular level. The author would earnestly request biophysicists to study in the future the spindle membrane based on the principle of mitosis.

2) Various phenomena manifested as a part of cell vitality and difficult to analyse biophysically at present

The problems belonging to this category are difficult at present to analyse because they are separate physical phenomena in the in vivo and in the in vitro conditions in the isolated state. These problems are: 1) The process of kinetochore arrangement of each chromosome in one plane at the equator of the metaphase spindle (Wada 1966); 2) The process of cell plate formation which appears at the beginning of telophase with the fibrils constituting the cell plate arranging themselves perpendicularly to those of the phragmoplast (Satô 1959); 3) The biochemical analysis of nucleolar RNA which is considered to play the role of a catalyser-like reaction for reversible folding and unfolding of the nuclear sap proteins (Wada 1977); 4) The influence of polarity in each cell which controls the arrangement of fibrous proteins at the formation of metaphase spindles.

All of these phenomena can be grasped and recognized in living mitotic cells by in vivo observations or under the polarization microscope. However, no effective techniques have yet developed to analyse these phenomena in biophysics or in biochemistry at present.

\section{Concluding remarks}

In the course of evolution of organisms, one of the procaryotes which would have aquired the genetic character and which would put the gene DNA together in one cavity might have become a predecessor of the eucaryotes (Wada 1976). 
Allsopp (1969) pointed out two major advances over the nuclear equivalent of the procaryotes: first, the possession of a nuclear envelope, and second, the distribution of the genetic material into discrete chromosomes.

In addition it is natural to consider that the appearance of a membrane bounded nucleus might have brought on the differentiation of karyoplasm and cytoplasm. In order to distribute gene DNA (chromosomes) equivalently into daughter nuclei, the karyokinesis would have been prepared by being equipped with a nuclear membrane in teleonomy under gene control.

With this viewpoint in consideration, the author has attempted to elucidate the mechanism of mitosis based on in vivo observations and experiments. The results of these studies on living mitotic cells have been published successively since 1930 (Wada 1950, 1966, 1977, etc.). The conclusion that karyokinesis is performed according to a common principle both in plant and animal cells (Wada 1977) have been reached. Unfortunately, the essentiality of in vivo observations, and the existence of the spindle membrane, for the elucidation of mitosis have not been recognized by most cytologists working on this subject. The author hasn't the least expectation of the elucidation to be achieved on the chromosome movement in anaphase by a) Inoué's dynamic equilibrium hypothesis, b) Bajer's zipper hypothesis and c) McIntosh's sliding filament theory as reported in 'Molecules and Cell Movement, (ed. Inoué and Stephens 1976) nor d) in Sato's assembly and disassembly hypothesis reported in 'Cell Motility' (ed. Hatano, Ishikawa and Sato 1979). These investigations disregarded many important findings and principles introduced by advances in biology, genetics, biophysics and molecular biology during the last half of this century as pointed out in the introduction to this paper.

The present author has experienced the ancient wisdom that truth must be simple and clear, yet since the establishment of cytology the mechanism of mitosis still remains an enigma in fixation cytology (including electron microscopic studies as well).

\section{Acknowledgement}

The author is indebted to Dr. T. Ino, Department of Genetics, The University of Tokyo, for valuable suggestions on genetics of molecular architechture and to Dr. A. Hanaoka, Jikei University, School of Medicine, for his help in preparation of the photographs. He also wishes to express sincere thanks to Prof. Daniel McCoy of the Science English Center, Faculty of Science and Technology, Sophia University Tokyo, for kind and continuous help in reading the manuscript.

\section{References}

Allsopp, A. 1969. Phylogenetic relationships of the procaryota and the origin of the eucaryotic cell. New Phytol. 68: 591-612.

Bajer, A. and Molè-Bajer, J. 1975. Lateral movement in the spindle and the mechanism of mitosis. In Molecules and Cell Movement (S. Inoué and R. E. Stephens eds.), pp: 77-96. Raven Press, New York.

Beadle, G. W. and Tatum, E. L. 1941. Genetic control of biochemical reactions in Neurospora. Proc. Natl. Acad. Sci. U.S.A. 27: 499-506. 
Fujii, K. 1931. Cytology, Past and Present. Iwanami, Tokyo. (in Japanese)

Ino, T. 1980. Genetics of Molecular Architechture. Baifu Kan, Tokyo. (in Japanese)

Inoué, S. 1953. Polarization optical studies of the mitotic spindle I. The demonstration of spindle fibers in living cells. Chromosoma $5: 487-500$.

- and Ritter, H. 1975. Dynamics of mitotic spindle organization and function. In Molecules and Cell Movement (S. Inoué and R. E. Stephens eds.), pp. 3-30. Raven Press, New York.

Izutsu, K., Sato, H., Aoki, N. and Nakabayashi, H. 1974. Cine-micrographic analysis of chromosome movements in meiotic divisions of grasshopper spermatocytes using a sensitive polarizing microscope. Symposium of Japan Society for Cell Biology 25: 140-145. (in Japanese)

Mazia, D. and Dan, K. 1952. The isolation and biochemical characterization of the mitotic apparatus of dividing cells. Proc. Natl. Acad. Sci. U.S.A. 38: 826-838.

McIntosh, J. R., Cande, W. Z. and Synder, J. A. 1975. Structure and physiology of the mammalian mitotic spindle. In Molecules and Cell Movement (S. Inoué and R. E. Stephens eds.), pp: 31-76. Raven Press, New York.

Monod, J. 1970. Chance and Necessary. Translated by A. Wainhouse. Vintage Books, New York.

Nakao, Y., Nakanishi, Y.H. and Wada, B. 1968. Karyokinetic studies and the behavior of centrioles in the spermatocytes meiosis of silkworm and grasshopper. Cytologia 33: $125-136$.

Naylor, E. 1926. The hydrogen ion concentration and the staining of sections of plant tissue. Amer. J. Bot. 13: 265-275.

Olah, L. V. and Bozzola, J. J. 1972. Effect of digitonin on cellular division IV. Interrelation between nucleus and phragmoplast. Cytologia 37: 365-376.

Sabatini, D. D., Rensh, K. and Barrnett, K. J. 1963. Cytochemistry and electron microscopy: the preservation of cellular ultrastructure and enzymatic activity of aldehyde fixation. J. Cell Biol. 17: 19-58.

Sakai, H., Hamaguchi, M., Kimura, I. and Hiramoto, Y. 1979. Chromosome motion in the isolated mitotic apparatus and comparison with in vivo anaphase. In Cell Motility: Molecules and Organization (Hatano, S., Ishikawa, H. and Sato, H. eds.), pp: 609-619. Univ. Tokyo Press, Tokyo.

Sato, H., Ohnuki, Y. and Sato, Y. 1979. Assembry and disassembly of the mitotic spindle. In Cell Motility: Molecules and Organization (Hatano, S., Ishikawa, H. and Sato, H. eds.), pp: 551-568. Univ. Tokyo Press, Tokyo.

Satô, S. 1958. Electron microscope studies on the mitotic figure I. Fine structure of the metaphase spindle. Cytologia 23: 383-394.

- 1959. Ditto II. Phragmoplast and cell plate. Cytologia 24: 98-106.

- 1960. Ditto III. Process of the spindle formation. Cytologia 25: 119-131.

Shimamura, T. 1940. Studies on the effect of centrifugal force upon nuclear division. Cytologia 11: 186-216.

Sprey, B. and Hasche, A. 1972. Ultrastrukturelle Veränderungen der Kernmembran von Lepidium sativum L. in der Mitose. Cytologia 37: 721-745.

Wada, Akiyoshi 1972. Dichroic spectra of biopolymers oriented by flow. Applied Spectroscopy Review 6: 1-30.

Wada, B. 1935. Mikrurugische Untersuchungen lebender Zellen in der Teilung II. Das Verhalten der Spindelfigur und einige ihrer physikalischen Eigenschaften in den somatischen Zellen. Cytologia 6: 381-406.

- 1943. Eine neue Methode zur Lebendbeobachtung der Mitose bei den Tradescantia-Haarzellen. Cytologia 13: 139-145.

- 1950. The mechanism of mitosis based on studies of submicroscopic structure and of the living state of the Tradescantia cell. Cytologia 16:1-26.

- and Fukunaga, K. 1957. A new technique to demonstrate the surface membrane of the spindle in preparations. Cytologia 22: 442-451.

- and Izutsu, K. 1961. Effects of ultraviolet microbeam irradiation on mitosis studied in Trades- 
cantia cells in vivo. Cytologia 26: 480-491.

-, Nagao, K. and Awasawa, M. 1963. Demonstration of spindle membrane in fixed preparations of root tip cells in Vicia faba and Allium cepa. Rep. Lib. Arts and Sci. Fac. Shizuoka Univ. (Nat. Sci.) 3: 185-192.

- and Kusunoki, F. 1964a. Spindle membrane in meiosis of pollen mother cells of Tradescantia and in mitosis of endosperm cells of Zephyranthes. Cytologia 29: 109-117.

- and - 1964b. Spindle membrane in mitosis of Ehrlich ascites tumor cells. Cytologia 28: 118-124.

-, Hanaoka, A. and Niitsu, T. 1964. Entity of the karyokinetic spindle in mitosis of sea urchin eggs. Cytologia 29: 443-452.

- 1966. Analysis of Mitosis. Cytologia 30 (Suppl. No.): 1-158.

- 1969. Mitotic cell studies based on in vivo observations II. New interpretations on behavior of the centriole-aster system. Cytologia 34: 484-495.

- 1970a. Ditto III. Biological significance of $9+2$ tubloid pattern, de novo appearance of blepharoplasts, disappearance of centrioles in higher plant cells and flagellum formation. Cytologia 35: 378-390.

- 1970b. Ditto IV. The raison d'être for the spindle membrane and the atractoplasm being composed of fibrils. Cytologia 35: 483-499.

- 1972. Ditto VI. Artifact problems of the mitotic figures under the electron microscope. Cytologia 37: 709-719.

- 1974. Ditto VII. The intrinsic nature of cancer cells and of carcinogenesis as interpreted from the viewpoint in reference to cell biology. Cytologia 39: 165-178.

- 1977. Ditto IX. A principle for the mechanism of the karyokinesis. Cytologia 42: 587-610.

- 1978. Ditto X. Problems on the objectivity of fixation cytology, of genetic information and of the teleonomy in the structure and function of the karyokinetic spindle. Cytologia 43 : 605-615.

- 1979. Cytology of carcinogenesis: The mechanism of unrestricted cell proliferation and of somatic cell mutation. Cytologia 44: 249-258.

Yasui, K. and Fujii, K. 1951. On the cell constituents and cell division with special reference to the staining reaction in buffer solution at $\mathrm{pH} 1.2$ to 8.0 . Cytologia 16: 131-152.

\section{Note added to the proof}

Recently, Hiramoto and Shôji (at the annual meeting of the Japan Society of Cell Biology held at Tokyo in November 1980) reported that in the eggs of the sand-dollar, Clypeaster japonicus, chromosome movement in anaphase took place in the spindle which was separated in vivo from the asters including the centrioles by compressing the eggs between a cover slip and a glass slide.

Wada would greatly like to estimate Hiramoto's experiment as an important example which gives evidence that the karyokinetic spindle in the case of animal cells is also independent of asters and centrioles in the cytoplasm. The separation of the karyokinetic spindles would not be achieved, if the nuclear membrane were to break down before spindle formation. 